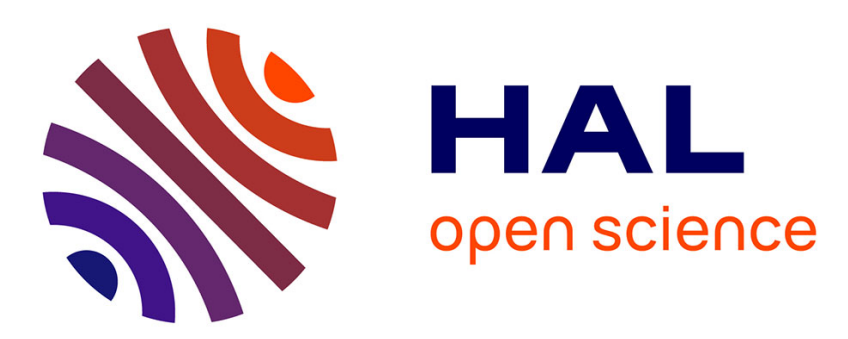

\title{
Médicaments du métabolisme, de l'allergologie, de la rhumatologie et de la neurologie
}

\author{
Jacques Buxeraud, Sébastien Faure
}

\section{To cite this version:}

Jacques Buxeraud, Sébastien Faure. Médicaments du métabolisme, de l'allergologie, de la rhumatologie et de la neurologie. Actualités Pharmaceutiques, 2019, 58, pp.37 - 44. 10.1016/j.actpha.2019.04.007 . hal-03486109

\section{HAL Id: hal-03486109 \\ https://hal.science/hal-03486109}

Submitted on 20 Dec 2021

HAL is a multi-disciplinary open access archive for the deposit and dissemination of scientific research documents, whether they are published or not. The documents may come from teaching and research institutions in France or abroad, or from public or private research centers.
L'archive ouverte pluridisciplinaire HAL, est destinée au dépôt et à la diffusion de documents scientifiques de niveau recherche, publiés ou non, émanant des établissements d'enseignement et de recherche français ou étrangers, des laboratoires publics ou privés.

\section{(ㄷ)(1) $\$$}

Distributed under a Creative Commons Attribution - NonCommerciall 4.0 International 
Dochead dossier

Sous-dochead L'essentiel sur les nouveaux médicaments à l'officine

\title{
Médicaments du métabolisme, de l'allergologie, de la rhumatologie et de la neurologie
}

\author{
Jacques Buxeraud ${ }^{\mathrm{a}, *}$ \\ Professeur émérite des Universités

\section{Sébastien Faure ${ }^{b}$} \\ Professeur des Universités
}

${ }^{a}$ Faculté de pharmacie, 2 rue du Docteur-Marcland, 87025 Limoges cedex, France

bUFR santé, Département pharmacie, Université d'Angers, 16 boulevard Daviers, 49045 Angers, France

\section{*Auteur correspondant.}

Adresse e-mail : jacques.buxeraud@unilim.fr (J. Buxeraud).

\section{Résumé}

Chaque année, des secteurs isolés de la thérapeutique bénéficient de l’arrivée de médicaments sur le marché. Un tout nouveau concept de traitement des hypercholestérolémies a vu le jour, alors que d'autres spécialités récentes en allergologie, rhumatologie et neurologie wsont le fruit d'innovations amorcées déjà depuis quelques années.

(C) 2019

Mots clés - asfotase alfa ; alirocumab ; evolocumab ; hypolipémiant ; hypophosphatasie ; sarilumab Summary à venir

(C) 2019

Keywords à venir 
Les anticorps monoclonaux investissent dorénavant le secteur des hypolipémiants avec l'alirocumab et l'évolocumab, tous deux inhibiteurs de la proprotéine convertase subtilisine/kexine de type 9 (PCSK9). Quant au traitement de la polyarthrite rhumatoïde (PR), il s'est enrichi d'un nouvel anticorps monoclonal inhibiteur de l'interleukine 6 (IL-6), le sarilumab. Par ailleurs, un nouveau médicament orphelin est disponible, l'asfotase alfa (Strensiq ${ }^{\circledR}$ ), indiqué dans le traitement enzymatique substitutif au long cours chez les patients atteints d'hypophosphatasie, ainsi qu'une immunothérapie allergénique $\left(\right.$ Acarizax $\left.^{\circledR}\right)$ qui est en fait le premier médicament sur la base d’extrait allergénique d'acariens administré par voie sublinguale.

T1 Un nouvel hypolipémiant, l’alirocumab

TEG1 Spécialité : Praluent ${ }^{\circledR}$ (alirocumab), Sanofi Aventis France.

TEG1 Présentation : solution injectable SC à $75 \mathrm{mg} / \mathrm{mL}$ et à $150 \mathrm{mg} / \mathrm{mL}$ (stylos préremplis de $1 \mathrm{~mL}$, boîtes de 2).

TEG1 Prescription et délivrance : médicament en liste I. Prescription initiale annuelle réservée aux spécialistes en cardiologie, en endocrinologie, diabète et maladies métaboliques ou en médecine interne. Renouvellement non restreint. Médicament d'exception.

TEG1 Remboursement Sécurité sociale à $65 \%$. Collectivités.

Amélioration du service médical rendu (ASMR) : IV en association à un traitement hypolipémiant optimisé, chez les patients adultes présentant une hypercholestérolémie familiale hétérozygote, insuffisamment contrôlée et nécessitant un traitement par LDL-aphérèse.

TEG1 Praluent ${ }^{\circledast}$ est indiqué chez l'adulte présentant une hypercholestérolémie primaire (hétérozygote familiale et non familiale) ou une dyslipidémie mixte, en complément d'un régime alimentaire soit :

- en association avec une statine seule ou une statine avec d'autres thérapies hypolipémiantes chez les patients ne pouvant atteindre leur objectif de LDL-cholestérol (LDL-C), sous statine à dose maximale tolérée ou,

- seul ou en association avec d'autres thérapies hypolipémiantes chez les sujets intolérants aux statines, ou chez qui les statines sont contre-indiquées.

TEG1 L'alirocumab est un anticorps monoclonal qui agit en se liant à la PCSK9 ${ }^{1}$. Il empêche ainsi la liaison de cette enzyme circulante aux récepteurs LDL présents à la surface de la cellule hépatique et évite leur dégradation. Le nombre de récepteurs disponibles pour capter le LDL-C circulant augmente, ce qui permet donc d'en diminuer le taux. Les récepteurs au LDL-C se lient également à des lipoprotéines de très basse densité (VLDL) riches en triglycérides et de densité intermédiaire (IDL). Ainsi, l'alirocumab permet une réduction des autres lipoprotéines [1]. 


\section{T2 Posologie}

TEG1 La dose initiale habituelle est de $\mathbf{7 5} \mathbf{~ m g}$ par voie SC une fois toutes les deux semaines. Chez les patients qui exigent une diminution plus importante du taux de LDL-C, le traitement peut démarrer à la dose de $150 \mathrm{mg}$ une fois toutes les deux semaines ou $300 \mathrm{mg}$ une fois toutes les quatre semaines. La posologie est ensuite ajustée individuellement, la dose maximale étant de $150 \mathrm{mg}$ toutes les deux semaines.

TEG1 Les modalités d'administration sont suivantes :

- l'injection se réalise dans la cuisse, l'abdomen ou le haut du bras ;

- pour la dose de $300 \mathrm{mg}$, il faut effectuer consécutivement deux injections de $150 \mathrm{mg}$ sur deux sites différents ;

- les sites doivent être alternés à chaque administration ;

- l'injection ne doit pas être réalisée dans des zones d'affections cutanées actives ou lésées (érythèmes solaires, éruptions cutanées, zones inflammatoires ou infectées) ;

- le produit ne doit pas être administré en même temps que d'autres médicaments injectables sur le même site d'injection.

Après une formation à la bonne technique dispensée par un professionnel de santé, l'injection de Praluent ${ }^{\circledR}$ peut être effectuée par le patient, un accompagnant ou un soignant.

\section{T2 Contre-indications}

Une hypersensibilité à la substance active ou à l'un des excipients contre-indique l'utilisation de Praluent $^{\circledR}$.

Ce médicament doit être utilisé avec prudence en cas d'insuffisance rénale ou hépatique sévère.

\section{T2 Effets indésirables}

TEG1 Les effets indésirables les plus fréquents ont été des réactions locales au site d'injection (érythème/rougeur, démangeaisons, gonflement, douleur/sensibilité), des symptômes des voies aériennes supérieures et un prurit.

TEG1 Des réactions allergiques générales, mais aussi rares et parfois graves, telles que I'hypersensibilité, l'eczéma nummulaire, l'urticaire et la vascularite d'hypersensibilité, ont été rapportées dans les études cliniques. 
T2 Précautions et surveillance du traitement

TEG1 Les paramètres lipidiques peuvent être évalués quatre à huit semaines après l'instauration ou l'ajustement posologique du traitement, la posologie pouvant alors être ajustée en fonction des résultats.

TEG1 Si des signes ou symptômes de réaction allergique grave se manifestent, Praluent ${ }^{\circledR}$ doit être arrêté et il convient d'instaurer un traitement symptomatique approprié.

\section{T2 Interactions médicamenteuses}

Alirocumab étant un agent biologique, aucun effet pharmacocinétique sur d'autres médicaments ni sur les enzymes du cytochrome P450 (CYP450) ne sont attendus.

\section{Encadré}

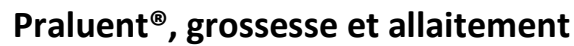

TEG1 Pendant la grossesse, l'utilisation de Praluent ${ }^{\circledR}$ n'est pas recommandée sauf si l'état clinique de la femme nécessite un traitement par l'alirocumab.

TEG1 Les effets de l'alirocumab sur les enfants allaités n'étant pas connus, il doit être décidé soit d'arrêter l'allaitement, soit d'interrompre le traitement par Praluent ${ }^{\circledR}$.

Respecter cet emplacement pour l'encadré / sur 1 colonne

T1 Un nouvel hypolipémiant, l'évolocumab

TEG1 Spécialité : Repatha ${ }^{\circledR}$ (évolocumab), Amgen

TEG1 Présentation : solution injectable (SC) à $140 \mathrm{mg}$ : stylo prérempli (SureClick) de $1 \mathrm{~mL}$ à usage unique (boîte unitaire).

TEG1 Prescription et délivrance : médicament en liste I. Prescription initiale annuelle réservée aux spécialistes en cardiologie, en endocrinologie, diabète et maladies métaboliques ou en médecine interne. Renouvellement non restreint.

TEG1 Médicament d'exception

TEG1 Remboursement Sécurité sociale à $65 \%$. Collectivités. 
TEG1 ASMR : IV chez l'adulte et l'adolescent à partir de 12 ans présentant une hypercholestérolémie héréditaire homozygote en association avec d'autres thérapies hypolipidémiantes.

TEG1 Repatha ${ }^{\circledR}$ est indiqué chez les patients présentant :

- une hypercholestérolémie primaire et une dyslipidémie mixte (adulte) ;

- une hypercholestérolémie familiale homozygote (adulte et à partir de 12 ans) ;

- une maladie cardiovasculaire athéroscléreuse établie (adulte avec infarctus du myocarde, accident vasculaire cérébral ou artériopathie périphérique) pour réduire le risque cardiovasculaire en diminuant le taux de LDL-C, en complément de la correction des autres facteurs de risque.

TEG1 L'évolocumab se lie de manière sélective à la PCSK9 et empêche sa forme circulante de se lier au récepteur des lipoprotéines de faible densité (LDLR) situé à la surface des cellules hépatiques, empêchant ainsi la dégradation du LDLR induite par la PCSK9. L'augmentation des taux de LDLR hépatiques se traduit ainsi par une diminution du LDL-C sérique (LDL-C).

\section{T2 Posologie}

TEG1 La posologie est en général de $\mathbf{1 4 0} \mathrm{mg}$ toutes les deux semaines ou $420 \mathrm{mg}$ une fois par mois, les deux doses étant cliniquement équivalentes. La dose de $140 \mathrm{mg}$ est administrée en utilisant un seul stylo prérempli et celle de $420 \mathrm{mg}$ en utilisant trois stylos préremplis dans un délai de 30 minutes.

TEG1 Repatha ${ }^{\circledR}$ est administré par voie SC dans l'abdomen, la cuisse ou le haut du bras. Les sites d'injection doivent être alternés et le produit ne doit pas être injecté dans des zones où la peau est sensible ou présente une ecchymose, une rougeur ou une induration.

TEG1 Le produit peut être auto-administré par le patient qui a reçu une formation adéquate.

\section{T2 Contre-indications}

Une hypersensibilité à la substance active ou à l'un des excipients contre-indique le traitement.

Repatha ${ }^{\circledR}$ doit être utilisé avec précaution chez les patients atteints d'insuffisance rénale ou hépatique sévère.

\section{T2 Effets indésirables}

Les effets indésirables le plus fréquemment rapportés au cours des essais pivots ont été une rhinopharyngite, une infection des voies respiratoires supérieures, dorsalgies, des arthralgies, la grippe et des réactions au site d'injection. 
T2 Interactions médicamenteuses

Aucune étude d'interaction médicamenteuse formelle n'a été réalisée avec Repatha ${ }^{\circledR}$.

\section{Encadré}

Repatha ${ }^{\circledR}$, grossesse et allaitement

TEG1 Pendant la grossesse, Repatha ${ }^{\circledR}$ ne doit pas être utilisé à moins que la situation clinique de la femme ne justifie un traitement avec évolocumab.

TEG1 II convient de décider d'arrêter l'allaitement ou d'interrompre/de s'abstenir du traitement avec Repatha ${ }^{\circledR}$, en prenant en compte le bénéfice de l'allaitement pour l'enfant au regard du bénéfice du traitement pour la femme.

Respecter cet emplacement pour l'encadré / 1 colonne

T1 Un médicament orphelin pour l'hypophosphatasie

TEG1 Spécialité : Strensiq ${ }^{\circledR}$ (asfotase alfa), Alexion Pharma France.

TEG1 Présentation : solution injectable à $40 \mathrm{mg} / \mathrm{mL}$ (flacons de $0,45 \mathrm{~mL}, 0,7 \mathrm{~mL}$ ou $1 \mathrm{~mL}$ en boîtes de 12) et à $100 \mathrm{mg} / \mathrm{mL}$ (flacon de $0,8 \mathrm{~mL}$ en boîte de 12 ).

TEG1 Prescription et délivrance : médicament en liste I, soumis à prescription hospitalière, nécessitant une surveillance particulière pendant le traitement. Ce médicament a reçu une autorisation de mise sur le marché (AMM) européenne, sous le statut de médicament orphelin.

TEG1 Remboursement Sécurité sociale à $65 \%$. Collectivités.

TEG1 ASMR : II.

TEG1 Strensiq ${ }^{\circledR}$ est indiqué dans le traitement enzymatique substitutif au long cours chez les patients atteints d'hypophosphatasie dont les premiers signes sont apparus avant l'âge de $\mathbf{1 8}$ ans pour traiter les manifestations osseuses de la maladie (encadré 1). Même si ce médicament est rarement délivré à l'officine, il est important de le connaître pour suivre l'avancée des recherches portant sur les maladies très rares.

TEG1 Le principe actif de Strensiq ${ }^{\circledR}$ est l'asfotase alfa, une glycoprotéine dont la structure chimique comprend l'ectodomaine de la phosphatase alcaline non spécifique de tissu et un fragment FC-décaaspartate possédant une activité enzymatique. L'asfotase favorise la minéralisation du squelette chez les patients atteints d'hypophosphatasie [2-4]. 


\section{Encadré 1. Connaître l'hypophosphatasie}

L’hypophosphatasie est :

- une maladie génétique rare, sévère et potentiellement fatale ;

- entraînée par une ou plusieurs mutations avec perte de fonction du gène codant pour la phosphatase alcaline non spécifique de tissu ;

- responsable de nombreuses manifestations osseuses incluant rachitisme/ostéomalacie, troubles du métabolisme phosphocalcique, troubles de la croissance et de la mobilité, insuffisance respiratoire pouvant nécessiter une ventilation mécanique et des crises convulsives répondant à la vitamine $\mathrm{B} 6$.

\section{Sur 1 colonne près de son appel}

\section{T2 Posologie}

TEG1 Le schéma posologique recommandé est de $\mathbf{2} \mathbf{~ m g / k g}$ par voie SC trois fois par semaine ou de $1 \mathrm{mg} / \mathrm{kg}$ par voie SC six fois par semaine. Le volume maximal de solution par injection ne doit pas excéder $1 \mathrm{~mL}$. Si un volume supérieur est nécessaire, plusieurs injections peuvent être effectuées simultanément. Les sites d'injection doivent être alternés et surveillés attentivement pour détecter des signes de réactions éventuelles.

TEG1 Les patients peuvent s'auto-injecter le médicament après avoir été correctement formés aux procédures d'administration. Le résumé des caractéristiques du produit (RCP) fournit un tableau très pratique qui précise les doses à administrer en fonction du poids corporel, le volume à injecter et le type de flacon à utiliser.

\section{T2 Durée du traitement}

Le traitement est prévu pour être maintenu jusqu'à la fin de la croissance. Sa poursuite au-delà de l'âge osseux adulte devra être discutée.

\section{T2 Contre-indications}

Une hypersensibilité sévère ou menaçant le pronostic vital à la substance active ou à l'un des excipients si l'hypersensibilité ne peut être contrôlée contre-indique l'utilisation de Strensiq ${ }^{\circledR}$. 
Les effets indésirables le plus fréquemment observés sont des réactions au site d'injection, des douleurs au niveau des extrémités, des myalgies et des réactions d'hypersensibilité comprenant : érythème/rougeur, pyrexie/fièvre, irritabilité, nausées, douleur, rigidité/frissons, hypoesthésie buccale, céphalées, bouffées vasomotrices et des signes et symptômes caractéristiques d'une anaphylaxie.

\section{Buxera_illus1}

\section{T2 Précautions et surveillance du traitement}

TEG1 Les réactions au site d'injection sont généralement correctement prévenues par l'alternance des sites d'injection. Toutefois, Strensiq ${ }^{\circledR}$ doit être interrompu chez tout patient présentant des réactions sévères.

TEG1 Des événements indésirables du type craniosynostose (pathologie en rapport avec une soudure prématurée d'une ou plusieurs sutures crâniennes), associée à une augmentation de la pression intracrânienne, comprenant également l'aggravation d'une craniosynostose préexistante, ont été rapportés chez des patients âgés de moins de 5 ans. Une surveillance régulière (incluant un examen du fond d'œil pour détecter des signes d'œdème papillaire) et une intervention rapide en cas d'hypertension intracrânienne sont recommandées dans cette population.

TEG1 Des cas de calcification oculaire (conjonctivale et cornéenne) et de néphrocalcinose ont été rapportés au cours des études cliniques. Un examen ophtalmologique et une échographie rénale doivent être réalisés régulièrement.

TEG1 Les concentrations sériques en parathormone peuvent augmenter plus particulièrement au cours des douze premières semaines de traitement. Il est conseillé de surveiller ces concentrations ainsi que la calcémie. Une supplémentation en calcium et en vitamine $D$ orale peut s'avérer nécessaire.

TEG1 Une prise de poids excessive peut être entraînée par le traitement, d'où la nécessite de surveiller le régime alimentaire du patient.

\section{T2 Interactions}

L'asfotase alfa contient un domaine catalytique de la phosphatase alcaline humaine non spécifique de tissu. Son administration interfère donc avec le dosage de routine de la phosphatase alcaline sérique Le médecin traitant doit signaler au laboratoire d'analyses que le patient est traité par un médicament qui modifie ses taux.

\section{Encadré}




\section{Strensiq ${ }^{\circledR}$, grossesse et allaitement}

La grossesse contre-indique la prise d'asfotase alfa qui n'est également pas recommandée chez les femmes en âge de procréer n'utilisant pas de contraception.

L'allaitement doit être interrompu au cours du traitement par l'asfotase alfa.

Respecter cet emplacement pour l'encadré / sur 1 colonne

T1 Un extrait allergénique à base d’acariens

TEG1 Spécialité : Acarizax ${ }^{\circledR}$ extrait allergénique standardisé d'acariens de la poussière de maison (Dermatophagoides pteronyssinus et Dermatophagoides farinae), ALK-Abelló.

TEG1 Présentation : lyophilisat oral à 12 SQ-HDM (boîte de 30) (encadré 2).

TEG1 Prescription et délivrance : médicament en liste I.

TEG1 Remboursement Sécurité sociale à $15 \%$. Collectivités pour l'adulte. Non remboursé par la Sécurité sociale et non agréé collectivités pour l'adolescent à la date du 19 janvier 2018.

TEG1 ASMR : V.

TEG1 Acarizax ${ }^{\circledR}$ est indiqué chez :

- les adultes (âgés de 18 à 65 ans) qui présentent une rhinite allergique aux acariens persistante modérée à sévère insuffisamment contrôlée par les traitements symptomatiques et/ou un asthme allergique aux acariens insuffisamment contrôlé par les corticostéroïdes inhalés et associé à une rhinite allergique légère à sévère aux acariens ;

- les adolescents (âgés de 12 à 17 ans) atteints d'une rhinite allergique aux acariens persistante modérée à sévère insuffisamment contrôlée par les traitements symptomatiques.

TEG1 Premier médicament à base d'extrait allergénique d'acariens administré par voie sublinguale, Acarizax ${ }^{\circledR}$ constitue une immunothérapie allergénique qui consiste en l'administration répétée d'allergènes à un individu allergique dans le but de modifier sa réponse immunitaire à leur encontre. L'activité pharmacodynamique de l'immunothérapie allergénique a pour cible le système immunitaire mais le mécanisme d'action exact à l'origine de l'effet clinique n'est pas totalement connu. Acarizax ${ }^{\circledR}$ agit sur la cause de l'allergie respiratoire aux acariens ; il induit une amélioration des symptômes et de la qualité de vie du patient (diminution du recours aux autres médicaments et réduction du risque d'exacerbations) [5-7].

Encadré 2. À propos de SQ-HDM 
SQ-HDM est l'unité de dose pour Acarizax ${ }^{\circledR}$ :

- SQ est une méthode de standardisation de la puissance biologique, de la teneur en allergènes majeurs et de la complexité de l'extrait allergénique ;

- HDM est l'abréviation de house dust mite (acarien de la poussière de maison).

Sur 1 colonne près de son appel

\section{T2 Posologie}

La posologie recommandée chez les adultes et les adolescents (âgés de 12 à 17 ans) est d'un lyophilisat oral (12 SQ-HDM) par jour. L'apparition de l'effet clinique est attendue huit à 14 semaines après l'instauration du traitement.

\section{T2 Durée du traitement}

Les recommandations thérapeutiques internationales préconisent une durée de traitement d'environ trois ans, mais s'il n'est pas observé d'amélioration pendant la première année de traitement par Acarizax $^{\circledR}$, sa poursuite n'est pas justifiée.

\section{T2 Contre-indications}

Les contre-indications au traitement par Acarizax ${ }^{\circledR}$ sont :

- hypersensibilité à l'un des excipients ;

- volume expiratoire maximal par seconde (VEMS) $<70 \%$ de la valeur théorique (après un traitement médicamenteux adapté) lors de l'initiation du traitement ;

- exacerbation sévère d'asthme au cours des trois derniers mois ;

- infection aiguë des voies respiratoires chez les sujets asthmatiques (la mise en route du traitement doit être différée jusqu'à sa guérison) ;

- maladies auto-immunes évolutives ou mal contrôlées, déficits immunitaires, immunodépression ou maladies néoplasiques malignes évolutives;

- inflammation buccale aiguë sévère ou plaies de la muqueuse buccale.

\section{T2 Effets indésirables}


TEG1 Les effets indésirables attendus sont la survenue de réactions allergiques locales légères à modérées au cours des premiers jours du traitement, disparaissant en un à trois mois. Dans la majorité des cas, elles apparaissent dans les cinq minutes suivant la prise et disparaissent en quelques minutes ou plusieurs heures. Des réactions allergiques oropharyngées plus sévères peuvent survenir.

TEG1 Des cas isolés d'aggravation aiguë sévère des symptômes d'asthme ont également été rapportés.

TEG1 En cas d'apparition d'effets indésirables significatifs liés à l'administration d'Acarizax ${ }^{\circledR}$, le recours à un traitement symptomatique de l'allergie doit être envisagé

\section{T2 Précautions et surveillance du traitement}

TEG1 En cas d'aggravation aiguë de l'asthme, les patients doivent consulter immédiatement un médecin. Acarizax ${ }^{\circledR}$ doit être initialement utilisé en complément du traitement antiasthmatique en cours et non en remplacement de celui-ci. Les médicaments administrés pour le contrôle de l'asthme ne doivent pas être arrêtés brutalement après l'instauration d'Acarizax ${ }^{\circledR}$. La diminution de leurs doses ne doit être envisagée que de façon progressive et sous contrôle médical selon les recommandations de prise en charge de l'asthme.

TEG1 Une réaction allergique systémique sévère, une exacerbation sévère d'asthme, un angioœdème, une dysphagie, une dyspnée, une dysphonie, une hypotension ou une sensation de constriction pharyngée imposent que le traitement soit interrompu. Un avis médical immédiat est requis. Les symptômes annonciateurs d'une réaction systémique peuvent inclure des flushs (bouffées vasomotrices), un prurit, une sensation de chaleur, un malaise général, une agitation et une anxiété. L'adrénaline peut être requise pour traiter ces manifestations.

TEG1 En cas d'inflammation sévère de la muqueuse buccale (lichen plan buccal, ulcérations ou mycose, par exemple), de plaies dans la bouche, de chirurgie buccopharyngée, y compris une extraction dentaire, ou la perte d'une dent, l'instauration du traitement par Acarizax ${ }^{\circledR}$ sera différée ou le traitement en cours temporairement interrompu, jusqu'à la cicatrisation.

TEG1 Des réactions allergiques locales sont attendues au cours du traitement par Acarizax ${ }^{\circledR}$ qui expose le patient aux allergènes auxquels il est allergique. En cas d'apparition de réactions indésirables locales significatives induites par son administration, l'utilisation d'un antihistaminique doit être envisagée.

TEG1 Des cas isolés d'œsophagite à éosinophiles ont été rapportés. Un avis médical est requis en cas de symptômes gastro-œsophagiens sévères ou persistants, tels qu'une dysphagie ou une dyspepsie.

TEG1 Des traces de protéines de poisson peuvent être retrouvées dans Acarizax ${ }^{\circledast}$. Les données disponibles n'ont pas mis en évidence de risque accru de réactions allergiques chez les patients présentant une allergie au poisson. Les données disponibles n'ont pas mis en évidence de risque accru de réactions chez les patients présentant une allergie au poisson. 
Acarizax $^{\circledR}$, grossesse et allaitement

TEG1 La grossesse contre-indique I'utilisation d'Acarizax ${ }^{\circledR}$. Si elle survient en cours de traitement, la décision de poursuivre ou non l'immunothérapie allergénique doit prendre en considération l'état clinique de la patiente ainsi que ses antécédents de réactions apparues lors des prises précédentes. En cas d'asthme préexistant, une surveillance étroite est recommandée pendant la grossesse.

TEG1 L'allaitement n'est pas déconseillé car il n'est pas attendu d'effets particuliers chez les enfants allaités.

Respecter cet emplacement pour l'encadré / sur 1 colonne

T1 Un immunosuppresseur pour la PR

TEG1 Spécialité : Kevzara ${ }^{\circledR}$ sarilumab, Sanofi Aventis France.

TEG1 Présentation : solution injectable SC à 150 mg et 200 mg (seringues et stylos préremplis, boîtes de 2).

TEG1 Prescription et délivrance : médicament en liste I. Médicament soumis à prescription initiale hospitalière annuelle. Prescription initiale et renouvellement réservés aux spécialistes en rhumatologie ou en médecine interne. Médicament nécessitant une surveillance particulière pendant le traitement. Médicament d'exception

TEG1 Remboursement Sécurité sociale à $65 \%$. Collectivités.

TEG1 ASMR : V.

TEG1 Kevzara ${ }^{\circledR}$ est indiqué en association au méthotrexate (MTX) chez les patients adultes atteints de PR.

TEG1 Cette PR doit répondre aux critères suivants : active, modérée à sévère ayant eu une réponse inadéquate ou intolérance à un ou plusieurs traitements de fond (DMARDs).

TEG1 Kevzara ${ }^{\circledR}$ peut être utilisé en monothérapie en cas d'intolérance au MTX ou lorsque le traitement avec le MTX est inadapté [8-10].

\section{T2 Un anticorps monoclonal inhibiteur de l'IL-6}

Kevzara ${ }^{\circledR}$ se lie de manière spécifique aux récepteurs solubles et membranaires de l'IL- 6 et inhibe la transmission du signal médié par les récepteurs de l'IL-6. Cette dernière joue un rôle majeur dans les symptômes de la PR comme la douleur, les articulations gonflées, la raideur matinale et la fatigue. 


\section{T2 Posologie}

TEG1 La posologie recommandée est de $\mathbf{2 0 0} \mathbf{~ m g}$ toutes les deux semaines, en injection SC. Une réduction à $150 \mathrm{mg}$ est recommandée en cas de neutropénie, de thrombopénie ou d'élévation des enzymes hépatiques,

TEG1 Le traitement doit être interrompu chez les patients qui développent une infection grave et ce, jusqu'à ce qu'elle soit contrôlée.

TEG1 L'instauration d'un traitement n'est pas recommandée chez les patients présentant :

- un nombre de neutrophiles inférieur à $2000 \mathrm{~mm}^{3}$;

- un nombre de plaquettes inférieur à $150000 / \mathrm{mm}^{3}$.

TEG1 Les sites d'injection (abdomen, cuisse et partie supérieure du bras) doivent être alternés à chaque administration et le produit ne doit pas être injecté si la peau est sensible, lésée ou présente des ecchymoses ou des cicatrices.

\section{T2 Contre-indications}

Le traitement est contre-indiqué dans les cas suivants :

- hypersensibilité à la substance active ou à l'un des excipients ;

- infections sévères actives.

II n'est pas recommandé chez les patients atteints d'une pathologie hépatique active ou d'une insuffisance hépatique.

\section{T2 Effets indésirables}

TEG1 Les effets indésirables le plus souvent rapportés au cours des études cliniques ont été : neutropénies, augmentation des taux d'alanine aminotransférase (ALAT), érythème au site d'injection, infections des voies aériennes supérieures et des voies urinaires.

TEG1 Les effets graves les plus fréquents ont été les infections (pneumonies, cellulite, infections opportunistes).

TEG1 Des cas de zona ont également été observés au cours des études cliniques. 
TEG1 Afin de détecter tout signe ou symptôme d'infection, les patients doivent être étroitement surveillés. Le traitement doit être interrompu en cas de survenue d'une infection grave ou opportuniste. Avant l'instauration du traitement, les facteurs de risque de la tuberculose doivent être évalués et un dépistage d'infection latente réalisé. Tout signe ou symptôme de la tuberculose doit être repéré, y compris chez les sujets dont le test de dépistage était négatif.

\section{TEG1 La surveillance biologique repose sur la mesure :}

- du nombre de neutrophiles et de plaquettes dans les quatre à huit semaines suivant le début du traitement, puis en fonction de l'évaluation clinique ;

- des taux d'ALAT et d'aspartate aminotransférase (ASAT) dans les quatre à huit semaines suivant I'instauration du traitement, puis tous les trois mois ; selon l'évaluation clinique, d'autres tests de la fonction hépatique, notamment le dosage de la bilirubine, peuvent être envisagés ;

- des paramètres lipidiques environ quatre à huit semaines après le début du traitement, puis environ tous les six mois.

TEG1 Les patients présentant des signes abdominaux d'apparition récente, tels qu'une douleur persistante associée à de la fièvre, doivent rapidement faire l'objet d'une évaluation. En effet, des cas de perforation gastro-intestinale ont été rapportés au cours des études cliniques.

TEG1 En cas de réaction d'hypersensibilité, les patients doivent consulter immédiatement un médecin.

TEG1 L'administration de vaccins vivants ou vivants atténués est proscrite pendant le traitement. Avant de le débuter, il est recommandé d'effectuer toutes les vaccinations nécessaires conformément aux recommandations vaccinales en vigueur.

\section{T2 Interactions médicamenteuses}

Plusieurs études ont montré que les cytokines et les modulateurs des cytokines pouvaient influencer l'expression et l'activité d'enzymes spécifiques du CYP450 (CYP1A2, CYP2C9, CYP2C19 et CYP3A4). La pharmacocinétique des médicaments co-administrés métabolisés par ces enzymes peut donc être altérée. La prudence est donc de mise avec les médicaments à indice thérapeutique étroit métabolisés par le CYP pour lesquels la posologie est ajustée individuellement : warfarine, théophylline, contraceptifs oraux, statines...

Encadré

Kevzara ${ }^{\circledR}$, femmes en âge de procréer, grossesse et allaitement 
TEG1 Les femmes en âge de procréer doivent utiliser une contraception efficace pendant le traitement et jusqu'à trois mois après son arrêt.

TEG1 La grossesse contre-indique l'utilisation de Kevzara ${ }^{\circledR}$, sauf si l'état clinique de la patiente exige un traitement par sarilumab.

TEG1 L'allaitement ou le traitement par sarilumab doit être interrompu, en considérant le bénéfice de l'allaitement pour l'enfant et le bénéfice du traitement pour la patiente.

Respecter cet emplacement pour l'encadré / sur 1 colonne

T1 Un nouvel antiparkinsonien

TEG1 Spécialité : Xadago ${ }^{\circledR}$ (safinamide), Zambon France.

TEG1 Présentation : comprimé pelliculé à 50 et à 100 mg (boîtes de 30).

TEG1 Prescription et délivrance : médicament en liste I.

TEG1 Non remboursé Sécurité sociale, collectivités.

TEG1 ASMR : V.

TEG1 Xadago ${ }^{\circledR}$ est indiqué chez des patients adultes atteints d'une maladie de Parkinson idiopathique fluctuant à un stade intermédiaire ou avancé de la maladie en association à une dose stable de Lévodopa (L-dopa) seule ou en association avec d'autres médicaments antiparkinsoniens [11-13].

TEG1 Xadago ${ }^{\circledR}$ est un médicament antiparkinsonien, inhibiteur hautement sélectif et réversible de monoamine-oxydase (IMAO) B, qui agit selon les deux mécanismes d'action, dopaminergique et non dopaminergique. Il entraîne une augmentation des niveaux extracellulaires de dopamine dans le striatum. Il est associé à l'inhibition voltage-dépendant des canaux sodiques $\left(\mathrm{Na}^{+}\right)$et à une modulation de la libération stimulée de glutamate.

Buxera_illus2

T2 Posologie

TEG1 Le traitement doit être démarré à la dose de $\mathbf{5 0 ~} \mathbf{~ m g}$ par jour, celle-ci pouvant être augmentée jusqu'à $100 \mathrm{mg} /$ jour selon la réponse clinique.

TEG1 La dose de $\mathbf{5 0} \mathbf{~ m g}$ par jour est recommandée chez les patients atteints d'insuffisance hépatique modérée. 


\section{T2 Contre-indications}

Xadago ${ }^{\circledR}$ est contre-indiqué dans les cas suivants :

- hypersensibilité à la substance active ou à l'un des excipients ;

- traitement concomitant avec d'autres IMAO ;

- association avec la péthidine ;

- insuffisance hépatique sévère ;

- chez les patients albinos, atteints de dégénérescence rétinienne, d'uvéite, de rétinopathie héréditaire ou de rétinopathie diabétique sévère évolutive.

\section{T2 Effets indésirables}

TEG1 L'effet indésirable qui a été le plus fréquemment rapporté est la dyskinésie. Elle peut survenir précocement au cours du traitement.

TEG1 Chez les patients traités par agonistes de la dopamine et/ou autres traitements de la dopamine, d'autres effets peuvent survenir : troubles du contrôle des impulsions, jeu pathologique, augmentation de la libido, hypersexualité, dépenses ou achats compulsifs, consommation excessive de nourriture et alimentation compulsive.

\section{T2 Précautions et surveillance du traitement}

TEG1 Un trouble du contrôle des impulsions (TCI) peut se manifester chez les patients traités par agonistes de la dopamine et/ou traitement dopaminergique. Certains cas de $\mathrm{TCl}$ ont également été observés avec d'autres IMAO. Le traitement par safinamide n'a pas été associé à une augmentation de la survenue de $\mathrm{TCl}$. Les patients et soignants doivent être informés des symptômes comportementaux associés au TCl qui ont été observés chez les sujets traités par IMAO (compulsions, pensées obsessionnelles, jeu pathologique, augmentation de la libido, hypersexualité, comportement impulsif et dépenses ou achats compulsifs, notamment).

TEG1 Les effets secondaires dopaminergiques peuvent être augmentés sous safinamide, qui est utilisé comme traitement adjuvant à la lévodopa. De même, toute dyskinésie préexistante peut être exacerbée, ce qui nécessite de diminuer les doses de la lévodopa.

\section{T2 Interactions médicamenteuses}

TEG1 Avec la tyramine alimentaire, $\mathrm{Xadago}^{\circledR}$ peut être utilisé en toute sécurité sans aucune restriction de celle-ci. 
TEG1 Avec les inhibiteurs sélectifs de la recapture de la sérotonine (ISRS), la safinamide doit être prescrite à la dose efficace la plus faible en surveillant l'apparition de symptômes sérotoninergiques.

TEG1 L'administration concomitante de Xadago $^{\circledR}$ avec la fluoxétine ou la fluvoxamine doit être évitée. Le cas échéant, ces médicaments doivent être utilisés à faibles doses. Une période de sevrage correspondant à cinq demi-vies de l'ISRS utilisé précédemment doit être envisagée avant l'instauration du traitement par safinamide.

TEG1 L'association avec les IMAO ou la péthidine est contre-indiquée. Un délai d'au moins sept jours doit être respecté entre l'arrêt de Xadago ${ }^{\circledR}$ et l'instauration de ce type de traitement.

TEG1 L'association avec le dextrométorphane n'est pas recommandée. Si un traitement concomitant est nécessaire, il appelle la prudence.

\section{Encadré}

\section{Xadago $^{\circledR}$, grossesse et allaitement}

TEG1 Chez les femmes en âge de procréer, $\mathrm{Xadago}^{\circledR}$ ne doit pas être administré sauf en cas d'utilisation d'un moyen de contraception efficace.

TEG1 La grossesse contre-indique l'administration de la safinamide.

TEG1 L'allaitement est proscrit.

\section{Respecter cet emplacement pour l'encadré}

\section{Note}

${ }^{1}$ La proprotéine convertase subtilisine/kexine de type 9 (PCSK9) est une enzyme, codée par le gène du même nom, situé sur le chromosome 1 humain, qui participe au métabolisme du cholestérol.

\section{Références}

[1] Paitraud D. Praluent solution injectable (alirocumab) : nouvel hypocholestérolémiant de la classe des PCSK9. Actualités Vidal, 22 février 2018.

www.vidal.fr/actualites/22584/praluent_solution_injectable_alirocumab_nouvel_hypocholesterole miant_de_la_classe_des_pcsk9/

[2] Haute Autorité de santé (HAS). Synthèse d'avis de la Commission de la transparence. Strensiq (asfotase alfa), enzymothérapie substitutive. Progrès thérapeutique important dans le traitement de I'hypophosphatasie. Mars 2016. www.has-sante.fr/portail/upload/docs/application/pdf/201606/strensiq_synthese_ct14864.pdf 
[3] Haute Autorité de santé (HAS). Commission de la transparence. Avis du 16 mars 2016. Asfotase alfa. www.has-sante.fr/portail/upload/docs/evamed/CT-

14864_STRENSIQ_PIC_INS_Avis3_CT14864.pdf

[4] Paitraud D. Strensiq (asfotase alfa) : première enzymothérapie substitutive dans le traitement de I'hypophosphatasie. Actualités Vidal, 5 février 2016.

www.vidal.fr/actualites/22564/strensiq_asfotase_alfa_premiere_enzymotherapie_substitutive_dans _le_traitement_de_I_hypophosphatasie/

[5] Cochois I. Acarizax lyophilisat oral : premier médicament à base d'extrait allergénique standardisé d'acariens. Actualités Vidal, 20 février 2018.

www.vidal.fr/actualites/22577/acarizax_lyophilisat_oral_premier_medicament_a_base_d_extrait_all ergenique_standardise_d_acariens/

[6] Haute Autorité de santé (HAS). Synthèse d'avis de la Commission de la transparence. Acarizax (extrait allergénique standardisé d'acariens Dermatophagoides pteronyssinus et Dermatophagoides farinae). Février 2017. www.has-sante.fr/portail/upload/docs/application/pdf/2017-

06/dir77/acarizax_synthese_ct15325.pdf

[7] Haute Autorité de santé (HAS). Commission de la transparence. Avis du 22 février 2017. Extrait allergénique standardisé d'acariens Dermatophagoides pteronyssinus et Dermatophagoides farinae. www.has-sante.fr/portail/upload/docs/evamed/CT-15325_ACARIZAX_PIC_INS_Avis3_CT15325.pdf

[8] Paitraud D. Polyarthrite rhumatoïde : Kevzara (sarilumab), nouvel inhibiteur des récepteurs d'IL6. Actualités Vidal, 4 octobre 2018.

www.vidal.fr/actualites/22886/polyarthrite_rhumatoide_kevzara_sarilumab_nouvel_inhibiteur_des_ recepteurs_d_il6/

[9] Haute Autorité de santé (HAS). Synthèse d'avis de la Commission de la transparence.

Kevzara (sarilumab), inhibiteur des récepteurs de l'interleukine 6. Janvier 2018. www.hassante.fr/portail/upload/docs/application/pdf/2018-04/kevzara_syntehse_ct16468.pdf

[10] Haute Autorité de santé (HAS). Commission de la transparence. Sarilumab. Avis du 10 janvier 2018. www.has-sante.fr/portail/upload/docs/evamed/CT-

16468_KEVZARA_PIC_INS_Avis2_CT16468.pdf

[11] European Medicines Agency (EMA). Xadago ${ }^{\circledR}$ safinamide.

www.ema.europa.eu/documents/overview/xadago-epar-summary-public_fr.pdf

[12] Haute Autorité de santé (HAS). Synthèse d'avis de la Commission de la transparence. Xadago safinamide (antiparkinsonien). Octobre 2015. www.has-

sante.fr/portail/upload/docs/application/pdf/2018-04/kevzara_syntehse_ct16468.pdf

[13] Haute Autorité de santé (HAS). Commission de la transparence. Xadago. Avis du 7 octobre 2015. www.has-sante.fr/portail/upload/docs/evamed/CT-14371_XADAGO_PIC_INS_Avis2_CT14371.pdf 
Déclaration de liens d'intérêts

Les auteurs déclarent ne pas avoir de liens d'intérêts.

\section{Illustrations}

\section{Buxera_illus1}

(C) adimas/stock.adobe.com

Strensiq ${ }^{\circledR}$ est indiqué dans le traitement enzymatique substitutif au long cours chez les patients atteints d'hypophosphatasie dont les premiers signes sont apparus avant l'âge de 18 ans pour traiter les manifestations osseuses de la maladie.

Sur 1 colonne près de son appel

\section{Buxera_illus2}

(C) peterschreiber.media/stock.adobe.com

Xadago ${ }^{\circledast}$ est un médicament antiparkinsonien, inhibiteur hautement sélectif et réversible de monoamine-oxydase $B$, qui entraîne une augmentation des niveaux extracellulaires de dopamine dans le striatum.

Sur 1 colonne près de son appel 


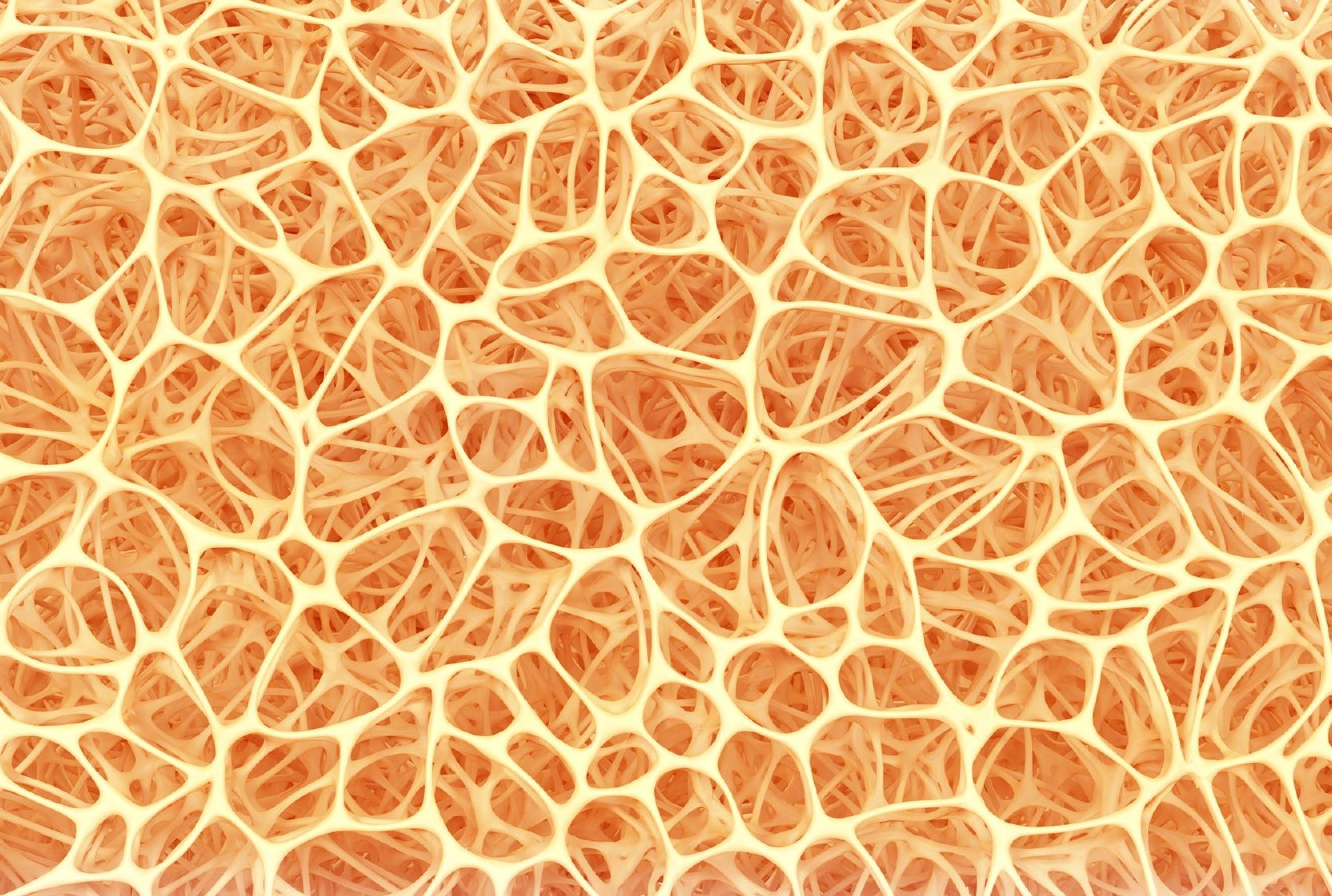




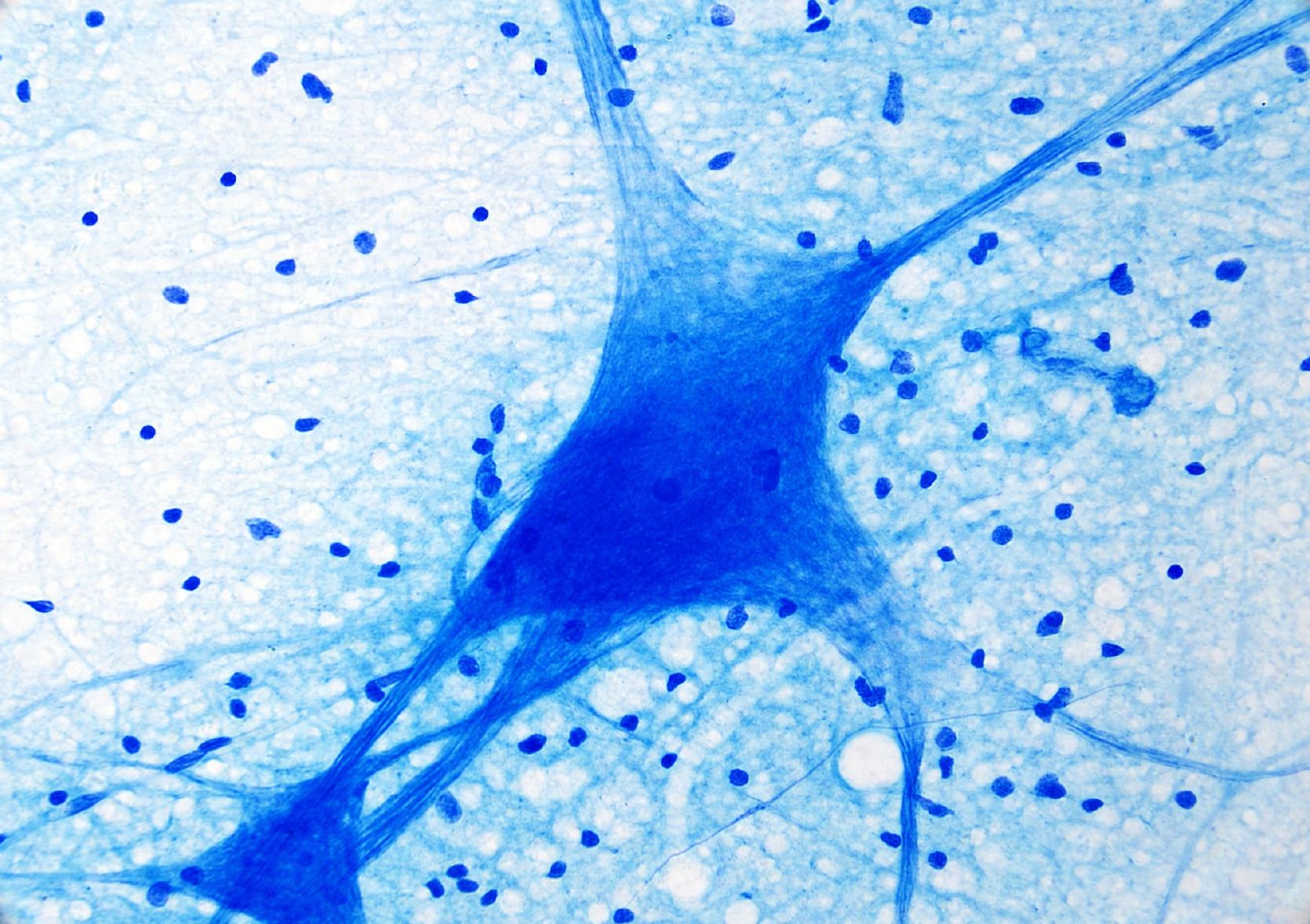

